\title{
Characterization of PVC-based polymer inclusion membranes with phosphonium ionic liquids
}

\author{
Katarzyna Witt $^{1}$ (D) . Elżbieta Radzymińska-Lenarcik ${ }^{1}$
}

Received: 15 October 2018 / Accepted: 11 October 2019 / Published online: 4 November 2019

(c) The Author(s) 2019

\begin{abstract}
Membrane techniques can be used to separate different compounds, i.e., toxic metal ions from waste waters. As any other method of separation, also this one, based on polymer inclusion membranes (PIMs), has certain limitations. One of them is the stability of membranes. In the present paper, the dependence of the physical and chemical properties on composition of PVC-based polymer inclusion membranes doped with phosphonium ionic liquids (CYPHOS IL 101, CYPHOS IL 104, CYPHOS IL 105 and CYPHOS IL 109) was studied. The thermal stability of investigated membranes was examined by thermogravimetry together with differential thermogravimetry analysis. Obtained results showed that studied PIMs are stable to the temperature of about $170^{\circ} \mathrm{C}$. The membrane morphology was examined by attenuated total reflectance Fourier-transform infrared (ATR-FTIR) spectroscopy, as well as scanning electron microscopy (SEM). The visibility of all characteristic bands in the ATR-FTIR spectra confirmed the presence of individual components in the membranes: a polymer, a plasticizer and the carriers. It also suggested that there were no signs of the covalent bond formation between the polymer, the plasticizer and the carrier. Only van der Waals or hydrogen bonds could be present. Moreover, in the SEM images of the investigated PIMs a rough surface without pores was observed.
\end{abstract}

Keywords Polymer inclusion membranes · Phosphonium ionic liquids · PVC · TG and DTG · SEM · FTIR

\section{Introduction}

Membrane techniques are effective and selective methods, because they can be used to separate different compounds, i.e., metal ions. A scope for their application and their environmental potential are extensive; thus, to this date they are the best alternative to known separation techniques. As any other method of separation, also this one, based on polymer inclusion membranes (PIMs), has certain limitations. One of them is the stability of membranes. To tackle this issue, numerous scientific studies are conducted. The key task is to select substrates making possible to obtain PIMs capable of selectively separating the studied substance with a high effectiveness while being characterized by a high mechanical strength. The main PIM components are: a polymer matrix, plasticizer and a metal ion carrier. Both polyvinyl chloride

Katarzyna Witt

Katarzyna.Witt@utp.edu.pl

1 Faculty of Chemical Technology and Engineering, UTP University of Science and Technology, Seminaryjna 3, PL 85326 Bydgoszcz, Poland
(PVC) [1] and cellulose triacetate (CTA) work well as the polymer matrix [2], while ethers [3] and adipates [4] are the most commonly used plasticizers. The compounds used as metal ion carriers include compounds from the imidazole [5, 6], $\beta$-diketones [7], or phosphonium ionic liquids [8] groups.

The phosphonium ionic liquids are characterized by their high thermal stability (ca. $400{ }^{\circ} \mathrm{C}$ ), and their temperature of decomposition mainly depends on an anion present in the ionic liquid structure. Deferm et al. [9] studied thermal stability of the commercially available trihexyl(tetradecyl) phosphonium chloride (CYPHOS IL 101). The authors of this paper proved that impurities present and higher heating rates lead to an under- and overestimation of the thermal stability, respectively. Moreover, the presence of oxygen leads to a lower thermal stability. Studies confirmed that by adding metal chlorides to the ionic liquid, the thermal stability is increased. The chloride anions are coordinated to the metal ion, so that the Lewis basicity of the anions is reduced.

Keating et al. [10] studied thermostability of CYPHOS IL 103 (trihexyl(tetradecyl)phosphonium decanoate) and CYPHOS IL 109. They found out that decomposition of these compounds in the air takes place in range of 
temperature from 200 to $475^{\circ} \mathrm{C}$. Black residues were found after heating to $740{ }^{\circ} \mathrm{C}$ in TG in 5.0 and 0.6 mass $/ \%$ for CYPHOS IL 103 and 109, respectively.

Properties other than thermal stability of selected phosphonium ionic liquids are shown in Table 1.

In recent years, the phosphonium ionic liquids have attracted a significant interest of researchers studying solvent extraction [11-14] and membrane techniques [15-18], due to their properties.

For example, Guo et al. [15] prepared a new type of polymer inclusion membrane (PIM), which was then applied in $\mathrm{Cr}(\mathrm{VI})$ transport. The novel PIMs are composed of three main components: poly(vinylidene fluoride) (PVDF), 1-alkyl-3-methylimidazolium hexafluorophosphate or tetrafluoroborate $\left(\mathrm{BF}_{4}\right)$ and trihexyl(tetradecyl)phosphonium bis2,4,4-(trimethylpentyl)phosphinate (CYPHOS IL 104). The authors proved that the permeability coefficient of membrane with CYPHOS IL 104 as carrier was 13 times higher than that of Aliquat 336. Baczyńska et al. [16] investigated the influence of PIMs containing CTA, o-nitrophenyloctyl ether (ONPOE), and of CYPHOS IL 101 or CYPHOS IL 104 aging on their morphology and the efficiency of $\mathrm{Zn}$ (II) transport. Pośpiech [17] used the same type of membranes for the facilitated transport of cadmium(II) and copper(II) ions from aqueous chloride solutions. She demonstrated that an increase in the carrier concentration results in an increase in the polymer membrane viscosity, and this forms an additional barrier in a diffusion mass transport through the PIM. Similar observations were made by Przewoźna et al. [18], who found that too high amount of the carrier could result in its secretion on the membrane surface, also forming an additional barrier to the mass transport through PIM.

It should be noted that all listed studies focused on membranes with CTA as the polymer matrix. The studies on membranes obtained from $\mathrm{PVC}$ or phosphonium ionic liquids are scarce. The polymer inclusion membranes with the PVC matrix and the CYPHOS IL 101 carrier were used for separation of $\mathrm{Zn}$ (II) ions from aqueous chloride solutions [19].

In the present paper, the dependence of the physical properties on composition of PVC-based membranes doped with phosphonium ionic liquids was investigated. The membranes were obtained by the solution casting method. The thermal stability of the membranes was examined by thermogravimetry (TG) together with differential thermogravimetry (DTG) analysis, while the membrane morphology was examined by attenuated total reflectance Fourier-transform infrared (ATR-FTIR) spectroscopy, as well as scanning electron microscopy (SEM).

\section{Experimental}

\section{Reagents}

The reagent, i.e., suspension polyvinyl chloride (PVC), of an average molecular weight of 72000 , was obtained from ANWIL, Wloclawek, Poland. The suspension PVC is formed during the industrial suspension polymerization of vinyl chloride (VCM). Tetrahydrofurane (analytical grade) was purchased from Avantor (Gliwice, Poland) and was used without further purification. The phosphonium ionic liquids (CYPHOS IL 101, CYPHOS IL 104, CYPHOS IL 105 and CYPHOS IL 109) and bis(2-ethylhexyl)adipate (DAO) were purchased from Sigma-Aldrich (Poznan, Poland).

\section{Membrane preparation and transport experiments}

A total of six different membranes were obtained. Blank membranes, PIM 1 and PIM 2, were prepared from a mixture of tetrahydrofurane with pure PVC and a mixture of tetrahydrofurane with PVC and DAO, respectively. Others membranes (PIM 3-PIM 6) were prepared from a ternary mixtures of tetrahydrofurane, containing PVC, DAO and one of phosphonium ionic liquid (Fig. 1).

Tetrahydrofurane was used only as a solvent. The obtained mixtures were poured out on ANUMBRA self-levelling dishes. After the evaporation of the tetrahydrofurane, $24 \mathrm{~h}$ later, the membranes were removed from the glass surface and immersed in distilled water for $12 \mathrm{~h}$ to obtain a film structure.

Compositions of the obtained membranes are presented in Table 2.

Table 1 Properties of chosen phosphonium ionic liquids

\begin{tabular}{lllll}
\hline & CYPHOS IL 101 & CYPHOS IL 104 & CYPHOS IL 105 & CYPHOS IL 109 \\
\hline Formula & $\mathrm{C}_{32} \mathrm{H}_{68} \mathrm{ClP}$ & $\mathrm{C}_{48} \mathrm{H}_{102} \mathrm{O}_{2} \mathrm{P}_{2}$ & $\mathrm{C}_{34} \mathrm{H}_{68} \mathrm{~N}_{3} \mathrm{P}$ & $\mathrm{C}_{34} \mathrm{H}_{68} \mathrm{~F}_{6} \mathrm{NO}_{4} \mathrm{PS}_{2}$ \\
Molecular mass & $519.31 \mathrm{~g} \mathrm{~mol}^{-1}$ & $773.27 \mathrm{~g} \mathrm{~mol}^{-1}$ & $549.90 \mathrm{~g} \mathrm{~mol}^{-1}$ & $764.00 \mathrm{~g} \mathrm{~mol}^{-1}$ \\
Flash point & $118{ }^{\circ} \mathrm{C}^{*}$ & $\mathrm{n} / \mathrm{d}$ & $\mathrm{n} / \mathrm{d}$ & $52{ }^{\circ} \mathrm{C}^{*}$ \\
Density & $0.895 \mathrm{~g} \mathrm{~mL}^{-1}$ at $20{ }^{\circ} \mathrm{C}^{*}$ & $0.895 \mathrm{~g} \mathrm{~mL}^{-1}$ at $20{ }^{\circ} \mathrm{C}^{*}$ & $0.900 \mathrm{~g} \mathrm{~mL}^{-1}$ at $20{ }^{\circ} \mathrm{C}^{*}$ & $1.070 \mathrm{~g} \mathrm{~mL}^{-1}$ at \\
& & & & $20{ }^{\circ} \mathrm{C}^{*}$ \\
Assay & $\geq 95.0 \%(\mathrm{NMR})^{*}$ & $\geq 95.0 \%(\mathrm{NMR})^{*}$ & $\geq 95.0 \%(\mathrm{NMR}) *$ & $\geq 95.0 \%(\mathrm{NMR}) *$ \\
\hline
\end{tabular}

*Information comes from the characteristic cards of the studied compounds 
Fig. 1 Structures of phosphonium ionic liquids (carriers in tested PIMs)<smiles>C[P+](C)(C)[Cl+]</smiles>

\section{CYPHOS IL 101}

trihexyl(tetradecyl)phosphonium chloride<smiles>CCCCC[P+](C)(C)[P+](C)(C)CC#N</smiles>

CYPHOS IL 105

trihexyl(tetradecyl)phosphonium dicyanamide<smiles>CC(CC(C)(C)C)CP(=O)([O-])CC(C)CC(C)(C)C</smiles>

CYPHOS IL 104

trihexyl(tetradecyl)phosphonium bis-2,4,4-(trimethylpentyl)phosphinate

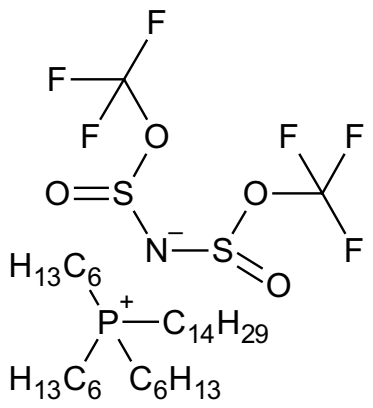

CYPHOS IL 109

trihexyl(tetradecyl)phosphonium bis(trifluoromethylsulfonyl)amide
Table 2 Composition of investigated membranes

\begin{tabular}{llll}
\hline Membrane & Polymer & Plasticizer & Carrier \\
\hline PIM 1 & PVC & - & - \\
PIM 2 & PVC & DAO & - \\
PIM 3 & PVC & DAO & CYPHOS IL 101 \\
PIM 4 & PVC & DAO & CYPHOS IL 104 \\
PIM 5 & PVC & DAO & CYPHOS IL 105 \\
PIM 6 & PVC & DAO & CYPHOS IL 109 \\
\hline
\end{tabular}

Membranes PIM 2-PIM 6 contained 15 vol\% of plasticizer, and membranes PIM 3-PIM 6 contained 20 vol\% of carrier

\section{PIMs characterization}

The obtained PIMs were characterized by thermogravimetry (TG), together with differential thermogravimetry (DTG) analysis, attenuated total reflectance Fouriertransform infrared (ATR-FTIR) spectroscopy, and scanning electron microscopy (SEM).

Thermal analyses (TG and DTG) of the PIMs were done in nitrogen atmosphere $\left(150 \mathrm{~cm}^{3} \mathrm{~min}^{-1}\right)$ using Netzsch TG $209 \mathrm{~F} 3$ Tarsus at the scanning speed of $10^{\circ} \mathrm{C} \mathrm{min}^{-1}$.
The ATR-FTIR spectra of the membranes were measured with a Bruker Alpha Spectrometer and were recorded within a wavenumber range of $450-4000 \mathrm{~cm}^{-1}$.

SEM images of the membranes were obtained with the Hitachi SU3500 SEM/EDS microscope operated at $10.0 \mathrm{kV}$. The membranes were visualized at $10.0 \times 10.0 \mu \mathrm{m}$ or $200.0 \times 200.0 \mu \mathrm{m}$ magnifications.

\section{Results and discussion}

The thermal stability, the presence of functional groups of individual substrates, and the analysis of surfaces of the obtained membranes were determined with TG and DTG, ATR-FTIR and SEM methods.

\section{TG and DTG analysis}

The thermal stability is understood as a temperature at which the chemical destruction of the polymer begins. Analyses of the thermal properties are used to determine the longterm life cycle of a given plastic, as well as to specify the maximum acceptable temperature of work. The thermal stability is closely related to the macromolecular structure. The higher stiffness of the macromolecule contributes to an 
PIM 1

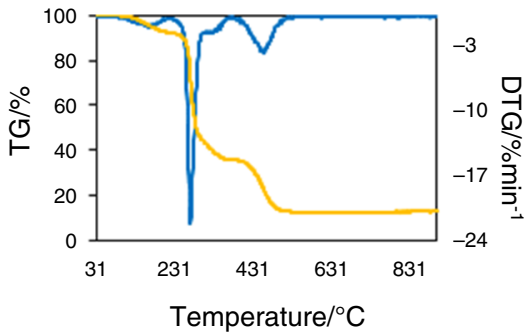

PIM 4

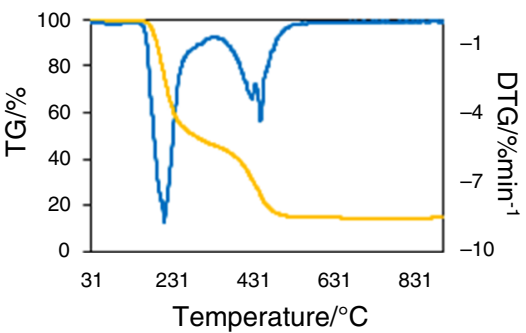

PIM 2

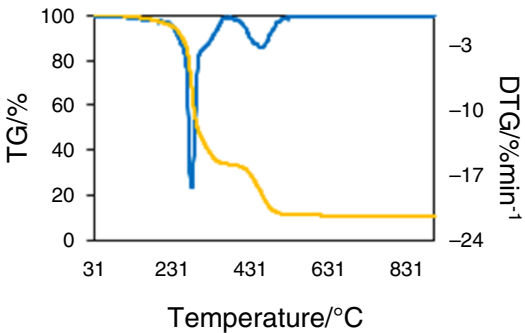

PIM 5

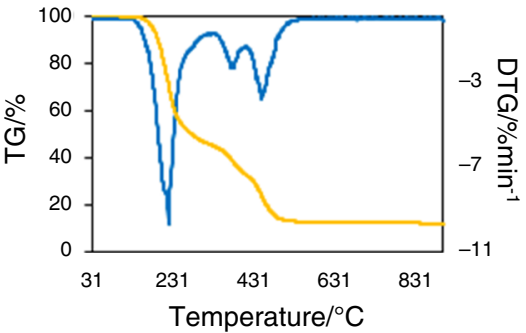

PIM 3

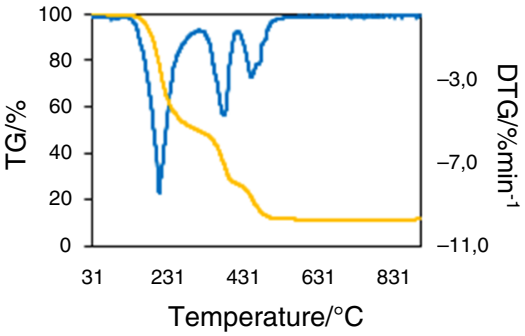

PIM 6

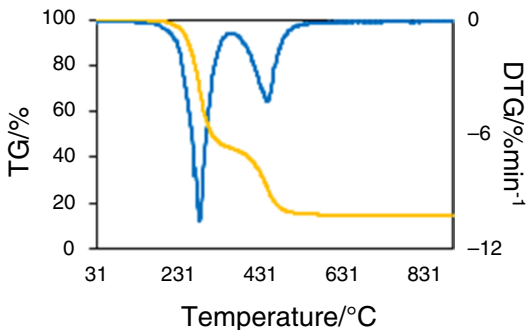

Fig. 2 TG and DTG curves of the investigated membranes

improvement in the thermal resistance. It can be increased, for example, by the presence of aromatic cycles, heterocycles, or hydrogen bonds.

The symmetry of the structure, the presence of substituents in the chains, and cross-linking are also of great importance. As in PIMs, the film stiffness is an unfavorable property; a plasticizer is added to the mixture, reducing the glass point and molecular interactions, and increasing polymer chain mobility, thus rendering the film flexible.
Studies on the polymer thermal resistance are usually conducted in the inert gas atmosphere. When polymers are heated to a high temperature, the chemical bonds in the main and the lateral chains are broken, low molecular gaseous products are generated, and, frequently, intramolecular reactions of cyclization and elimination occur. In the linear polymers, a depolymerization, i.e., reconstruction of a monomer, may also take place.

Figure 2 presents TG and DTG curves for the tested membranes.
PIM 1

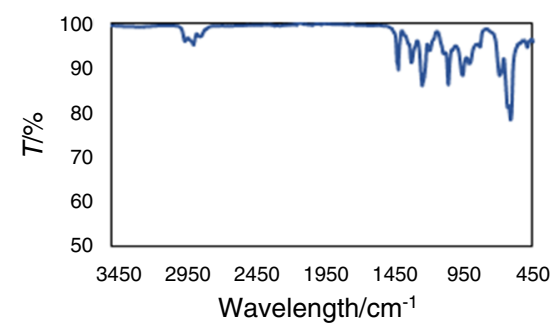

PIM 4

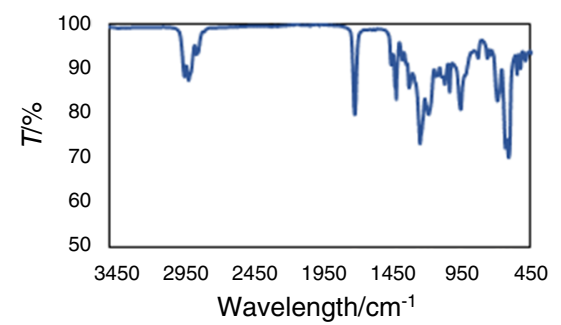

PIM 2

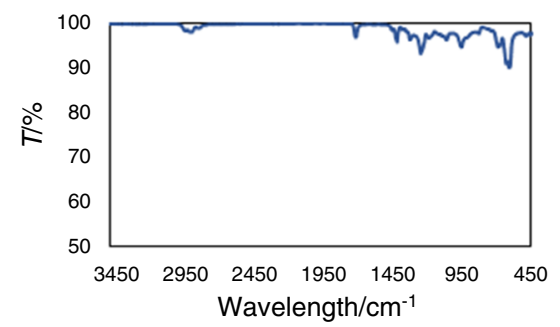

PIM 5

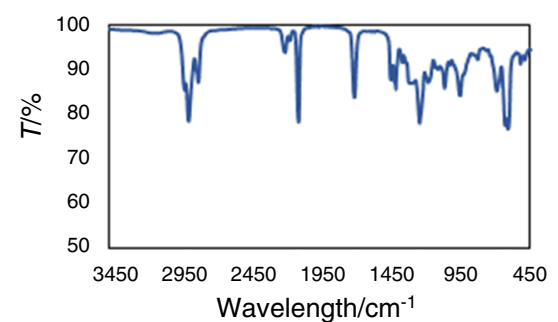

PIM 3

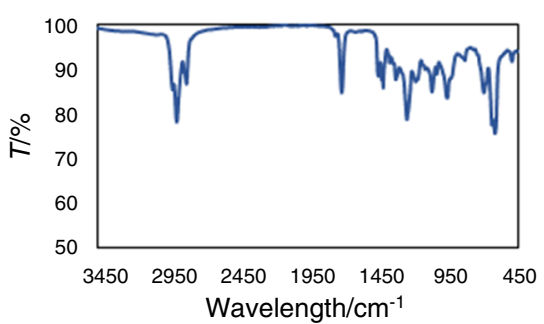

PIM 6

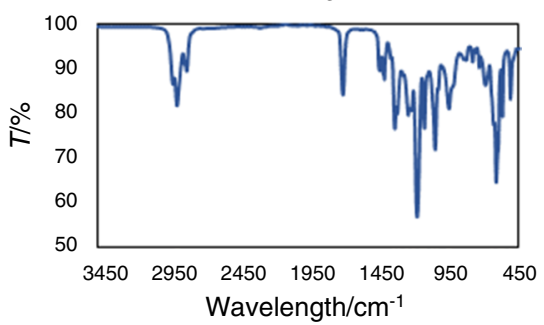

Fig. 3 ATR-FTIR spectra of the tested membranes 
Fig. 4 SEM images of tested membranes
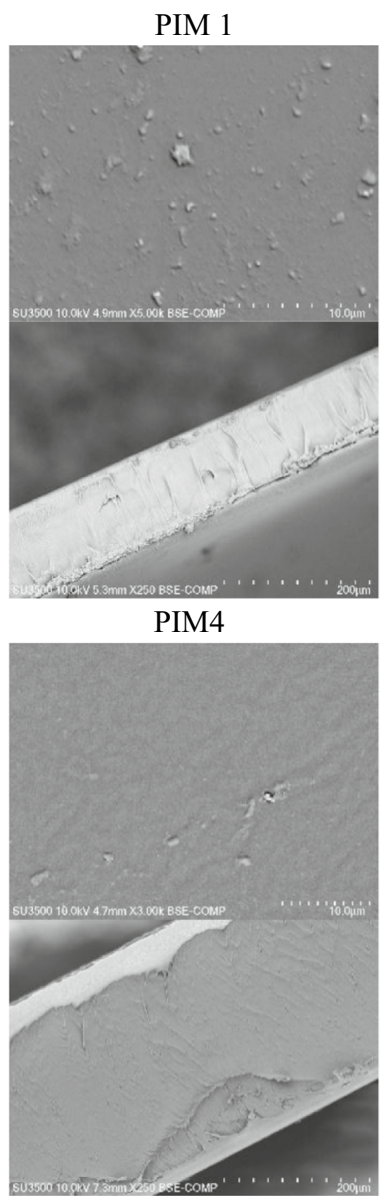

The curves shown in Fig. 2 demonstrate that when the plasticizer is added to pure PVC (PIM 1) at a level of $15 \mathrm{vol} \%$, the thermal stability of a membrane obtained this way (PIM 2) will not change significantly. The results indicate that the thermal decomposition of these two membranes can be divided into two stages. At the first stage, occurring in the range of $230-360{ }^{\circ} \mathrm{C}, \mathrm{HCl}$ is eliminated from polymer chain, and mass loss is $65 \%$. The second stage occurs in the range $390-520^{\circ} \mathrm{C}$, and the mass loss is $25 \%$. In these conditions, condensation and cross-linking of the resetting polyene take place $[20,21]$. Above $520{ }^{\circ} \mathrm{C}$, total charring of the membranes occurs.

An addition of the carrier to the membrane causes a relevant decrease in its thermal stability. Decomposition of the samples containing CYPHOSs (PIM 3-PIM 6) begins at ca. $170{ }^{\circ} \mathrm{C}$, and in most cases can be divided into three stages. Using of different phosphonium ionic liquids causes differences in the thermal distribution of the studied membranes. This is related to the various structure and thermal stability of the used CYPHOSs. The obtained results of the thermal analysis, as well as the visual evaluation of the membranes, indicate that the carrier plays a function of a plasticizer in the same way.

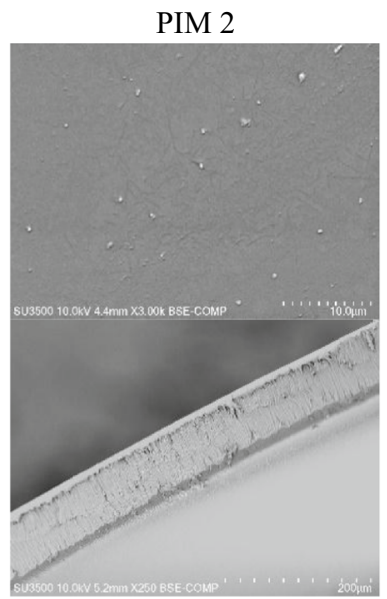

PIM 5

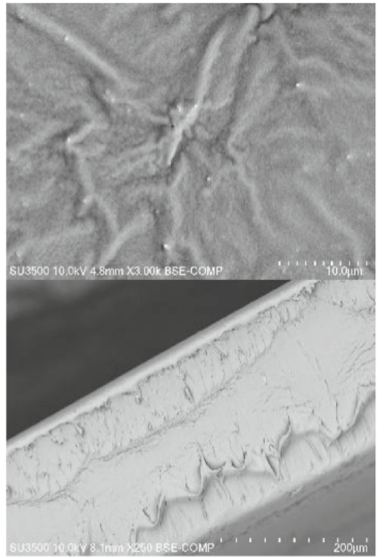

PIM 3

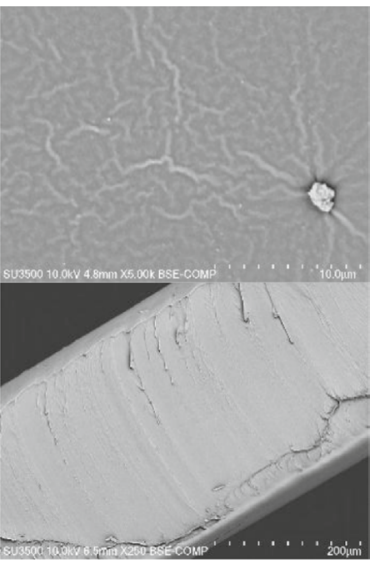

PIM 6

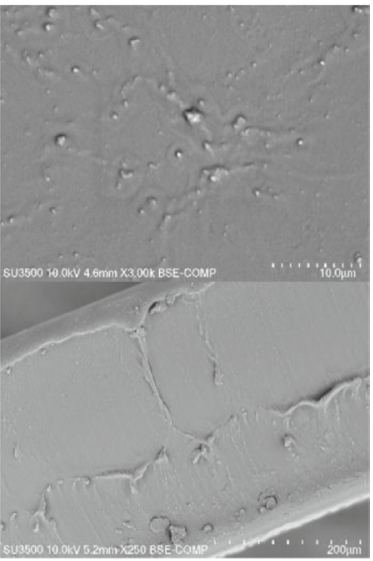

Studied PIMs are stable to the temperature of about $170{ }^{\circ} \mathrm{C}$.

\section{ATR-FTIR analysis}

The ATR-FTIR spectra of the tested membranes are shown in Fig. 3. The interpretation of infrared spectra was made using IRPal 2.0 program.

The pure PVC membrane spectrum (PIM 1) showed that bands at 612 and $690 \mathrm{~cm}^{-1}$ were attributed to the bending modes of the $\mathrm{C}-\mathrm{H}$ bonds, the bands at 1254 and $1335 \mathrm{~cm}^{-1}$ corresponded to the stretching modes of the $\mathrm{C}-\mathrm{X}$ bonds (where $\mathrm{X}$ is $\mathrm{Cl}$ ), and the band at $1426 \mathrm{~cm}^{-1}$ and the bands from 2850 to $3000 \mathrm{~cm}^{-1}$ corresponded to the stretching modes of the $\mathrm{C}-\mathrm{H}$ bonds.

In the PIM 2 spectrum (PVC + DAO membrane), the intensities of the bands corresponding to PVC were lower. Moreover, in this spectrum, an additional band was visible at $1725 \mathrm{~cm}^{-1}$. It corresponded to the stretching mode of the $\mathrm{C}=\mathrm{O}$ bond in the DAO molecule.

Characteristic bands in the range from 2850 to $2960 \mathrm{~cm}^{-1}$, attributed to the $-\mathrm{CH}_{2}$-and $-\mathrm{CH}_{3}$ bonds in the each carrier, are visible in the spectra for membranes from PIM 3 to PIM 7. 
The spectrum for PIM 4 contained characteristic bands from 1100 to $1200 \mathrm{~cm}^{-1}$, attributed to the $\mathrm{P}=\mathrm{O}$ bonds.

The $\mathrm{C} \equiv \mathrm{N}$ bond gives a characteristic band in the range of the wavelength from 2100 to $2200 \mathrm{~cm}^{-1}$. This band is visible in the spectrum of the PIM 5 membrane and confirms the presence of CYPHOS IL 105 in the tested film.

In the next spectrum (PIM 6), a $\mathrm{S}=\mathrm{O}$ bond gives a band at $1190 \mathrm{~cm}^{-1}$ and there are numerous bands in the range of $1000-1350 \mathrm{~cm}^{-1}$, confirming the presence of $\mathrm{C}-\mathrm{F}$ bonds in the membrane.

The visibility of all characteristic bands in the above ATRFTIR spectra confirmed the presence of individual components in the membranes: a polymer-PVC, a plasticizer-DAO and the carriers-phosphonium ionic liquids (CYPHOS IL 101, CYPHOS IL 104, CYPHOS IL 105, CYPHOS IL 109). It also suggested that there were no signs of the covalent bond formation between the polymer, the plasticizer, and the carrier. Only van der Waals or hydrogen bonds could be present.

\section{SEM analysis}

Scanning electron microscopy (SEM) was used to determine the appearance of the membrane surfaces and cross sections. The obtained images are shown in Fig. 4.

The surface of PIM of pure PVC contains lumps, and this may indicate incomplete melting of the polymer (PIM 1). Other membranes, containing 15 vol\% of plasticizer, had more dense structures (PIM 2-PIM 6). Moreover, in the SEM images of the surface and the cross section of the investigated PIMs no pores were found. The obtained results are consistent with observations of Ugur [22], who also did not find any pores in PIM when analyzing SEM images shown at the $\mu \mathrm{m}$ scale.

The images of the membranes containing carriers (PIM 3-PIM 6) showed a clear roughness of the film surfaces. In the case of PIM 5, the roughness was much more visible. Carriers could crystallize in the membrane and, for example, CYPHOS IL 109 molecules migrated to the membrane surface, causing its roughness.

\section{Conclusions}

The thermal stability of polymers is closely related to the macromolecular structure. It can be increased by the presence of aromatic cycles, heterocycles, or hydrogen bonds. Obtained results of TG and DTG analyses of tested PIMs showed that when the plasticizer is added to pure PVC at a level of $15 \mathrm{vol} \%$, the thermal stability of a membrane obtained this way will not change significantly. An addition of the carrier to the membrane causes a relevant decrease in its thermal stability. Decomposition of the samples containing CYPHOSs begins at ca. $170{ }^{\circ} \mathrm{C}$.
The ATR-FTIR analysis confirmed the presence of individual components in the membranes: a PVC, a DAO, and the carriers-phosphonium ionic liquids. Results also suggested that there were no signs of the covalent bond formation between the polymer, the plasticizer, and the carrier. Only van der Waals or hydrogen bonds could be present. Moreover, the SEM analysis indicated a rough surface of tested PIMs. For example, the CYPHOS IL 109 molecules migrated to the membrane surface, causing an increase in its roughness.

Acknowledgements The new developments presented above were carried out within the 2007-2013 Innovative Economy Operational Programme, Sub-action 1.3.2., Support of the protection of industrial property generated by scientific entities as a result of R\&D works within Project No. UDA-POIG.01.03.02-04-077/12-01, financed by the European Regional Development Fund (ERDF) (85\% of co-financing) and from a designated subsidy ( $15 \%$ of co-financing).

Open Access This article is distributed under the terms of the Creative Commons Attribution 4.0 International License (http://creativeco mmons.org/licenses/by/4.0/), which permits unrestricted use, distribution, and reproduction in any medium, provided you give appropriate credit to the original author(s) and the source, provide a link to the Creative Commons license, and indicate if changes were made.

\section{References}

1. Raut DR, Mohapatra PK. A novel PVC based polymer inclusion membrane containing TODGA as the extractant for the preconcentration of americium from acidic feed solutions. Sep Sci Technol. 2013;48(16):2499-505. https://doi.org/10.1080/01496 395.2013.807837.

2. Benavente J, Romero V, Vázquez MI, Anticó E, Fontàs C. Electrochemical characterization of a polymer inclusion membrane made of cellulose triacetate and Aliquat 336 and its application to sulfonamides separation. Separations. 2018;5(5):1-12. https ://doi.org/10.3390/separations5010005.

3. Ulewicz M, Walkowiak W. Removal of $\mathrm{Zn}(\mathrm{II}), \mathrm{Cd}(\mathrm{II})$ and $\mathrm{Pb}(\mathrm{II})$ using polymer inclusion membrane transport with proton ionizable DB-16-C-5 crown ethers. Physicochem Probl Miner. 2006;40:185-94.

4. Witt K, Radzyminska-Lenarcik E, Kosciuszko A, Gierszewska M, Ziuziakowski K. The influence of the morphology and mechanical properties of polymer inclusion membranes (PIMs) on zinc ion separation from aqueous solutions. Polymers. 2018;10(2):1-14. https://doi.org/10.3390/polym 10020 134.

5. Radzyminska-Lenarcik E, Ulewicz M. The use of 1-alkylimidzoles for selective separation of zinc ions in the transport process across a polymeric inclusion membrane. Physicochem Probl Miner. 2014;50(1):131-42. https://doi.org/10.5277/ppmp1 40112.

6. Lu Y, Cao Y, Lu Y, Yang T. Thermal stability and lifetime of [AMIM]Cl-PFSA composite membranes. J Therm Anal Calorim. 2017;128(32):1601-15. https://doi.org/10.1007/s 1097 3-016-6065-7.

7. Witt K, Radzymińska-Lenarcik E, Urbaniak W. Selective transport of zinc ions through a novel polymer inclusion membranes (PIMS) containing $\beta$-diketone derivatives as a carrier reagents. Sep Sci Technol. 2016;51(15-16):2620-7. https://doi.org/10.1080/01496 395.2016.1167084. 
8. Baczyńska M, Regel-Rosocka M. Phosphonium ionic liquids as carriers of metal ions. Przem Chem. 2013;92(9):1574-6.

9. Deferm C, Van den Bossche A, Luyten J, Oosterhof H, Fransaer J, Binnemans K. Thermal stability of ionic liquid Cyphos IL 101. Phys Chem Chem Phys. 2018;20:2444-56. https://doi. org/10.1039/c7cp08556g.

10. Keating MY, Gao F, Ramsey JB. TGA-MS study of the decomposition of phosphorus-containing ionic liquids trihexyl(tetradecyl) phosphonium decanoate and trihexyltetradecylphosphonium bis[(trifluoromethyl)sulfonyl] amide. J Therm Anal Calorim. 2011;106:207-11. https://doi.org/10.1007/s10973-011-1528-3.

11. Zhua Z, Tulpatowicz K, Pranoloa Y, Cheng C. Solvent extraction of molybdenum and vanadium from sulphate solutions with Cyphos IL 101. Hydrometallurgy. 2015;154:72-7. https://doi. org/10.1016/j.hydromet.2015.03.005.

12. Singh R, Mahandra H, Gupta B. Optimization of a solvent extraction route for the recovery of Mo from petroleum refinery spent catalyst using Cyphos IL 102. Solvent Extr Ion Exch. 2018;36(4):401-19.

13. Marták J, Schlosser Š. Phosphonium ionic liquids as new, reactive extractants of lactic acid. Chem Pap. 2006;60(5):395-8. https:// doi.org/10.2478/s11696-006-0072-2.

14. Kumari A, Sinha MK, Sahu SK, Pandey BD. Solvent extraction and separation of trivalent lanthanides using Cyphos IL 104, a novel phosphonium ionic liquid as extractant. Solvent Extr Ion Exch. 2016;34(5):469-84. https://doi.org/10.1080/07366 299.2016.1207459.

15. Guo L, Liu Y, Zhang Ch, Chen J. Preparation of PVDF-based polymer inclusion membrane using ionic liquid plasticizer and Cyphos IL 104 carrier for Cr(VI) transport. J Membr Sci. 2011;372(1-2):314-21. https://doi.org/10.1016/j.memsc i.2011.02.014.

16. Baczyńska M, Słomka Ż, Rzelewska M, Waszak M, Nowicki M, Regel-Rosocka M. Characterization of polymer inclusion membranes (PIM) containing phosphonium ionic liquids and their application for separation of $\mathrm{Zn}$ (II) from Fe(III). J Chem Technol Biotechnol. 2018;93(6):1767-77. https://doi.org/10.1002/ jctb.5552.
17. Pośpiech B. Application of phosphonium ionic liquids as ion carriers in polymer inclusion membranes (PIMs) for separation of cadmium(II) and copper(II) from aqueous solutions. J Solut Chem. 2015;44(12):2431-47. https://doi.org/10.1007/s1095 3-015-0413-2.

18. Przewoźna M, Gajewki P, Michalak N, Bogacki MB, Skrzypczak A. Determination of the percolation threshold for the oxalic, tartaric, and lactic acids transport through polymer inclusion membranes with 1-alkylimidazoles as a carrier. Sep Sci Technol. 2014;49:1745-55. https://doi.org/10.1080/01496395.2014.90646 4.

19. Kogelnig D, Regelsberger A, Stojanovic A, Jirsa F, Krachler $\mathrm{R}$, Keppler BK. A polymer inclusion membrane based on ionic liquid triheksyl(tetradecyl)phosphonium chloride and PVC for solid-liquid extraction on Zn(II) from hydrochloric acid solution. Monatsh Chem. 2011;142:769-72. https://doi.org/10.1007/s0070 6-011-0530-6.

20. Jiménez A, Berenguer V, López J, Sánchez A. Thermal degradation study of poly(vinyl chloride): kinetic analysis of thermogravimetric data. J Appl Polym Sci. 1993;50:1565-73. https://doi. org/10.1002/app.1993.070500910.

21. Li G, Wang M, Huang X, Li H, He H. Effect of zinc maleate/ zinc oxide complex on thermal stability of poly(vinyl chloride). J Appl Polym Sci. 2015;132(7):41464-72. https://doi.org/10.1002/ app.41464.

22. Ugur A, Sener I, Hol A, Korkmaz Aploguz H, Elci L. Facilited transport of $\mathrm{Zn}(\mathrm{II})$ and $\mathrm{Cd}$ (II) ions through polymer inclusion membranes immobilized with a calix[4]resorcinarene derivative. J Macromol Sci A. 2014;51:611-8. https://doi.org/10.1080/10601 325.2014 .924833

Publisher's Note Springer Nature remains neutral with regard to jurisdictional claims in published maps and institutional affiliations. 